\title{
Experimental Investigation of Effects of Magnetic Water on Germination and Growth Rate of Coriander (Coriandrum sativum) in ex-vitro Conditions
}

\author{
Luwin Tzib, and Dion Daniels*
}

Department of Science, Faculty of Science and Technology

University of Belize, Hummingbird Avenue Belmopan, Cayo District, Belize C.A.

\begin{abstract}
A magnetic field can be macroscopic in nature (current in a wire) or microscopic (current due to electrons in atoms) and it affects all living organisms since it is naturally a component of our environment known as the geomagnetic field. Various researches have shown that a plant's overall growth and development is affected either positively or negatively by a magnetic field. Research has also shown that magnetic fields can also influence plant's growth by applying it directly or indirectly. A cost-effective method of applying an indirect magnetic field to a plant is by magnetic water treatment, which is done by exposing ordinary water to a magnetic field and then irrigating the plants. The objective of this research was to determine the effects of magnetic water on the germination and growth rate of coriander in ex vitro conditions. There were 11 different treatments in this experiment including the control, with five different magnetic field strengths and two different exposure times for each magnetic field strength. The germination rate was observed and recorded after two weeks, while growth rate measurements were made starting from the third week after planting. Results showed that magnetic water treatment did not influence germination, but had a positive effect on the growth rate of coriander.
\end{abstract}

Key words: Magnetic field, Magnetic water treatment, Coriander, Germination.

\section{INTRODUCTION}

Magnetism has been a central phenomenon since the early rise of civilization. Natural magnetic phenomena was first reported by the ancient Greeks who described that the mineral lodestone (magnetite) had the ability to naturally attract iron filings and pieces of the same mineral [1]. Research in the last decade have shown very important results in the use of magnetic materials for the development of biomedicine, magnetic resonance imaging, robotics, data storage among others.

Magnetic treatment and its effects on plant health and development have been discussed for centuries. Some researchers have described magnetic fields (MF) as a "mysterious force" for plant growth which shows that the effect of magnetism on plant growth is not yet understood well. Magnetic fields are constant in the environment and the physical effects of it is just now being understood [2]. Electromagnetic fields (EMF) and MFs are believed to enhance seed vigor by enhancing the biochemical processes and by stimulating the activity of proteins and enzymes.

Research has shown that biological systems are not indifferent to a MF. It has been shown that plants exhibit physiological phenomena when brought into contact with MFs. External application of a MF or an EMF affects the growth and development of plants under both ex vitro and in vitro conditions [3]. An indirect way to apply a MF to plants is magnetic water (MW) which is water that has been treated with a MF. Water is a vital component to the development and survival of plants since it aids in photosynthesis, growth and respiration. Water acts as a transport system for plants by transporting dissolved nutrients, amino acids and sugars as it flows through the plant. Water is paramagnetic meaning that its molecules can be weakly attracted by a magnetic field, it holds a magnetic charge. Water has a dipole moment since it shares its electrons unequally between its atoms, which is why it is paramagnetic [1].

Various studies have shown that magnetic treatment of irrigation water improves the productivity of the water. MFs affect the structure of water and hydrated ions along with the physical-chemical properties and behavior of inorganic salts found in the water. The application of an external MF alters the chemical and electronic structure of the water. Changes occur to its solidifying 
and boiling point, the formation of clustering structures from linear and ring hydrogen-bound chains of molecules, these changes are all described by [4].

Magnetic water treatment (MWT) has many potential applications in agriculture and the environment. In nonmagnetic water, the water molecules are loosely attracted, this weak attraction influences pollutants and toxins to travel inside the molecule cluster [5]. Some of the smaller clusters that have toxins can enter the cell and have harmful effects. The MF breaks down the minerals in the water making them more bioavailable to the plant's cell. The result being a plant that is fully hydrated and has the adequate amount of minerals which then leads to better yield, earlier maturation time and healthier plants. Hydrating a plant with nonmagnetic water requires large volumes of water. Magnetic treatment of water restructures the water molecules into smaller clusters with each made up of six symmetrical organized molecules. These small clusters have hexagonal structures so it can easily enter the passageways in plant cell membranes [5].

Studies have shown that MW improves water quality, crop yield and induces seed germination. The biological effects of MW depend on the strength and exposure period of the MF or EMF to the water, along with its ion content, quality and its volume. Irrigating plants with MW accelerates its growth and it is influenced by various characteristics and specification of the treatment that the water has been exposed to. MWT also affects the soil-water-plant system. Irrigating the soil with MW increases the availability of phosphorous content compared to irrigation with nonmagnetic water [5]. Previous studies have shown that the effects of MWT varies depending on the plant type and the irrigation water used. There were statistically significant increases in plant yield and water productivity. MWT of recycled water and saline water increased celery yield respectively by $12 \%$ and $23 \%$ and water productivity by $12 \%$ and $24 \%$ [5]. MWT allows for lessening the amount of irrigation water, pesticides and fertilizers making it an eco-friendly practice in agriculture by using less resources. The objective of this research is to test the effect of magnetic water on germination and growth rate of coriander in ex-vitro conditions.

\section{MATERIALS \& METHODS}

The experiment was conducted at the University of Belize, Faculty of Science and Technology, Cayo District, Belize C.A. Two types of magnets, neodymium $(\mathrm{NdFeB})$ and ceramic, were used to conduct the research. All magnets utilized in this research were ordered online using both eBay and amazon. There were four treatments including neodymium magnets (bar, cube, disc, sphere) and one treatment including ceramic magnets (bar). The magnetic field strength (B) was calculated in Tesla (T) for each magnet using the equations below by [6]:

- Equation for block/bar magnets

$$
\mathrm{B}=\frac{B_{r}}{\pi}\left[\tan ^{-1}\left(\frac{L W}{2 z \sqrt{4 z^{2}+L^{2}+W^{2}}}\right)-\tan ^{-1}\left(\frac{L W}{2(D+z) \sqrt{4(D+z)^{2}+L^{2}+W^{2}}}\right)\right]
$$

- Equation for cylinder magnets

$$
\mathrm{B}=\frac{B_{r}}{2}\left(\frac{D+z}{\sqrt{R^{2}+(D+z)^{2}}}-\frac{z}{\sqrt{R^{2}+z^{2}}}\right)
$$

- Equation for bar magnets

$$
\mathrm{B}=B_{r} \frac{2}{3} \frac{R^{3}}{(R+z)^{2}}
$$

This experiment studied the effect of magnetic water on the germination and growth rate of coriander. Normal water was treated with a MF at different times ( $10 \& 20$ minutes) to produce magnetic water. Treatment of normal water was done by placing 2 magnets around recycled plastic containers at the North, South, East and West side of the container for a total of eight magnets for each treatment. The containers were then filled with water and was allowed to remain in the MF for 10 and 20 minutes for each treatment as shown in Table 1. 
International Journal of Advances in Scientific Research and Engineering (ijasre), Vol 8 (2), February -2022

Table 1. Different treatments for normal water along with the different time subjected to the magnetic field strengths

\begin{tabular}{|c|c|c|c|}
\hline Treatments & Magnetic Field Strength (T) & Type of Magnet & $\begin{array}{c}\text { Exposure Time } \\
\text { (minutes) }\end{array}$ \\
\hline 1 & Control & $-\cdots---$ & 0 \\
\hline 2 & $2.48 \times 10^{-3}$ & NdFeB (sphere) & 10 \\
\hline 3 & $2.48 \times 10^{-3}$ & NdFeB (sphere) & $\mathbf{2 0}$ \\
\hline 4 & $5.75 \times 10^{-2}$ & Ceramic (bar) & 10 \\
\hline 5 & $5.75 \times 10^{-2}$ & Ceramic (bar) & $\mathbf{2 0}$ \\
\hline 6 & $\mathbf{0 . 1 7 5}$ & NdFeB (disc) & 10 \\
\hline 7 & $\mathbf{0 . 1 7 5}$ & NdFeB (disc) & $\mathbf{2 0}$ \\
\hline 8 & $\mathbf{0 . 4 6 5}$ & NdFeB (bar) & 10 \\
\hline 9 & $\mathbf{0 . 4 6 5}$ & NdFeB (bar) & $\mathbf{2 0}$ \\
\hline 10 & $\mathbf{0 . 6 5 1}$ & NdFeB (cube) & 10 \\
\hline 11 & $\mathbf{0 . 6 5 1}$ & & 20 \\
\hline
\end{tabular}

Coriander seeds were obtained and a viability test was conducted prior to the onset of the experiment. The viability of the seed were found out to be $90 \%$. At the onset of the experiment 11 seedling trays (Figure 1) were obtained, one for each treatment with each seedling tray containing 50 cells. Potting material (Flower \& Vegetable Garden Mix) was obtained and moistened, which was then used to fill the trays. One randomly chosen coriander seed was planted in each cell of the seedling trays at a depth of $1.25 \mathrm{~cm}$ at the center of each cell. The seeds were then irrigated daily with its corresponding magnetic water treatment $(500 \mathrm{ml}$ for each tray). The germination rate was observed and recorded after two weeks and the growth rate was observed and recorded on the following week with recordings being taken weekly thereafter.

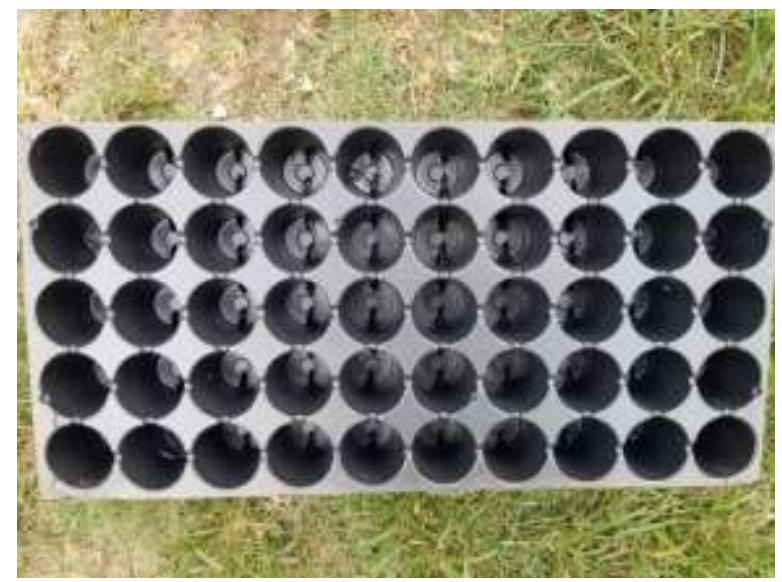

Figure 1. Seedling tray used to plant the coriander seeds

Data (plant height) for the growth rate were obtained for six weeks. Data analysis was done using SPSS (Statistical Package for Social Sciences). Microsoft Excel was used to create bar graphs to visually observe the differences in germination and growth rate of coriander between the different treatments. Prior to any analysis a Shapiro Wilk's test was conducted to check if the data collected was normally distributed. The means were then plotted on a bar graph using Microsoft excel. This was done to observe the effect of MF strength against germination and growth rate, exposure time against germination and growth rate and type of magnet against germination and growth rate.

An Analysis of Variance (ANOVA) was done to determine if there was a difference between the mean germination rate for the control and the 10 other treatments when looking at the effects of the MF strength, exposure time and the type of magnet. The ANOVA was followed by a Post Hoc test namely Tukey, this was used to determine which treatments were significantly different from the control and from each other. This process was also done for the growth rate. 


\section{RESULTS \& DISCUSSION}

Magnetic water (MW) has the potential to improve early seedling growth and increase nutrient content of seeds. Irrigation with MW has shown to increase some growth parameters, quantity, quality and chemical components of plants. MW is being used more frequently to increase crop production since it is not harmful on the environment and it is recommended as a way to save water.

\subsection{Germination Rate}

Figure 2 shows the effects of the different types of magnet, the strength and time to produce the magnetic water on the germination percentage of coriander seeds. The treatment with $\mathrm{NdFeB}$ (sphere 20) had a negative impact on the germination rate with significant difference when compared to the other treatments including the control. Treatments of $\mathrm{NdFeB}(\mathrm{Disc} 10)$ and Ceramic (Bar 20) had the highest germination percentages of $98 \%$ and $96 \%$ respectively. However, this showed no significant differences compared to the control, which indicates that for germination, there is not a need to use magnetized water. There were also no difference in terms of the time to magnetize the water.

There was a tendency that as the magnetic strength increases, the germination rates also increased up to a certain point and thereafter the percentages started to fall. However, these were minor differences noticed in the germination rate without any significant differences. MF treatment higher than $500 \mathrm{mT}$ is considered an abiotic stress factor that induces stress response in plant system [7]. This was the case in this research since after the MF strength got stronger, the stress factor in this case lowered the germination rate, however; without any significant statistical differences.

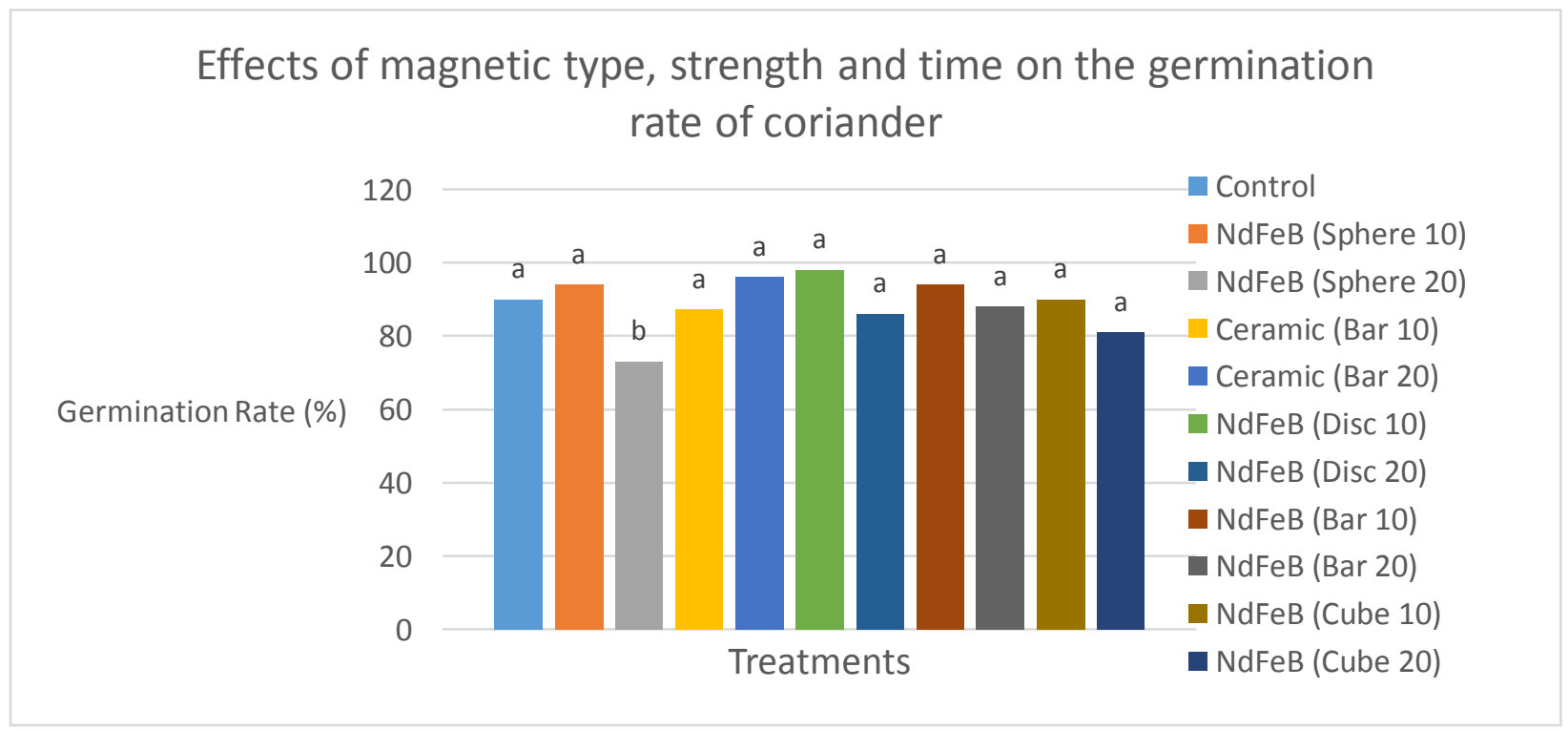

Different letters between treatments differ statistically for $p<0.05$ according to Tukey's test.

Figure 2. Germination rate of coriander two weeks after planting

Figure 3 took into account the average time that normal water was placed in the MF to make MW. This analysis did not take into account the MF strength. All the data for germination rate that used MW of 10 minutes were placed together and the means obtained with the same being done for 20 minutes and these means were plotted along with the mean germination rate of the control. From figure 3 it can be seen that the treatment that used MW of 10 minutes had the highest germination rate and the 20 minutes having the lowest germination rate. However, there was no significant statistical differences between the treatments and the control. 


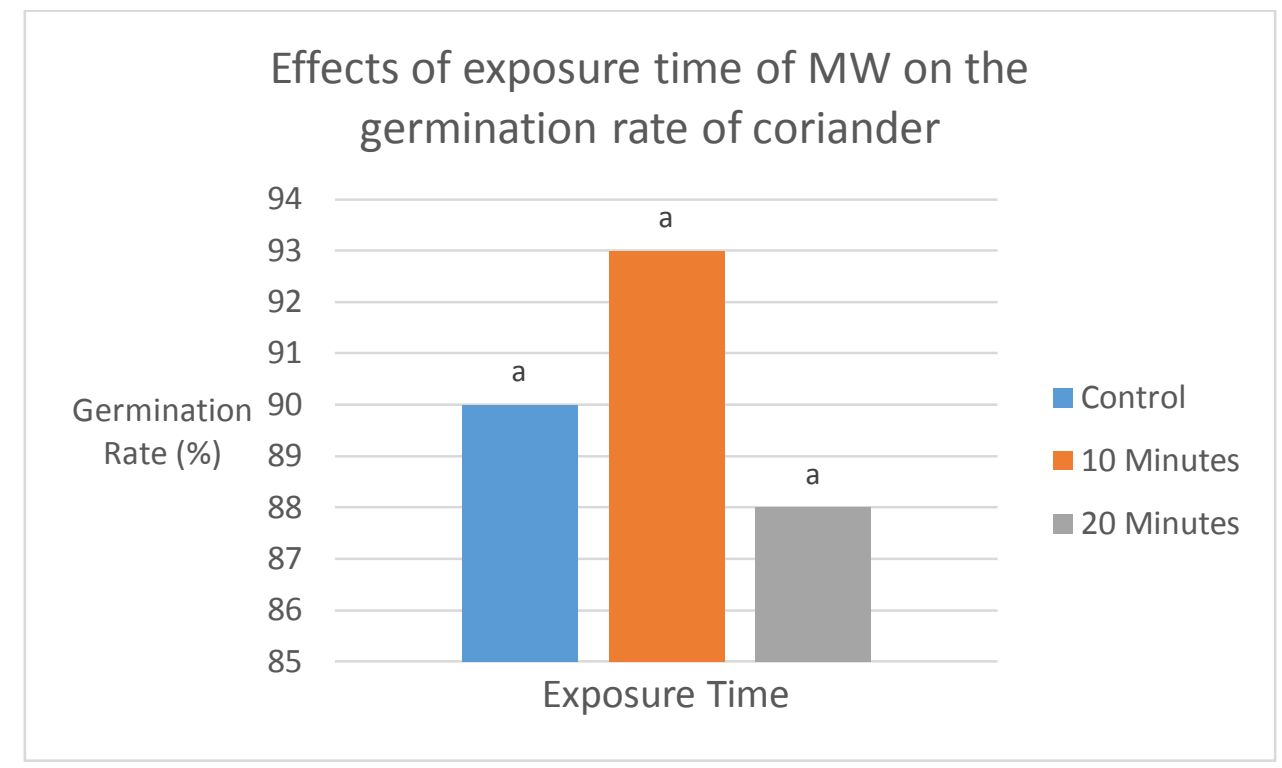

Different letters between treatments differ statistically for $p<0.05$ according to Tukey's test

Figure 3. Germination rate of coriander against different exposure times

Magnetic field strengths can retard the germination rate of plants [8]. Their research showed that MWT induced a negative effect in most of the indicators characterizing the germination rate of sunflower. Increasing exposure time to a MF led to a decrease in the germination rate of lemon balm seeds [9]. This is consistent with the results obtained in this research as shown in figure 3 where the 10 minute exposure time had the greatest germination rate. The lowest germination rate was obtained for the seeds that involved MWT [8].

All conditions that were tested against the germination rate of coriander showed that no condition had a statistically better germination rate or a negative germination rate than the control. One of the reasons that may have led to these results is that the effects of MW is strongly species and genotype dependent. Research has shown that MW affects germination rate in other species of plants such as different cultivars of wheat where the seeds were dipped and imbibed in MW exposed to $0.3 \mathrm{~T}$. These treatments had an increase in germination percentage of $9 \%$ to $30 \%$ higher in seven cultivars, but had a decrease in the germination rate of the remaining cultivars [3].

There has been many researches done on the influence of magnetic water on seed germination, but results are not always easy to compare since different conditions are being studied. The researchers all agree that the effect of MW depends on the plant species, genotype, ion content of the water, exposure time to MF, MF strength and other factors. There are results that show that MW influence plant germination while there are others that indicate that MW has no effect whatsoever on the germination rate of plants [10].

\subsection{Growth Rate}

Figure 4 shows the mean growth rate of coriander plotted against the MF strength of the different magnets used to make MW disregarding the time used to make it. It is noted that the highest growth rate was achieved at a magnetic field strength of $5.75 \mathrm{x}$ $10^{-2} \mathrm{~T}$ with significant difference compared to the other treatments. The lowest growth rate was obtained for $0.175 \mathrm{~T}$.

The highest growth rate observed can be attributed to the fact that this was a ceramic magnet, which has a weaker MF strength than $\mathrm{NdFeB}$ magnets and it has a different composition. This leads to the idea that MWT utilizing ceramic magnets might be a better option than $\mathrm{NdFeB}$ magnets, but further research must be done. The lowest growth rate was at a strength of $0.175 \mathrm{~T}$, which was a disc magnet. Shape decides where MF lines are denser which in turn determines the MF strength at different parts of the MF. It can be noted that the two lowest mean growth rate were observed with the sphere magnets and the disc magnets, which strengthens the idea that "circular" magnets have a detrimental effect on the growth rate of coriander. 


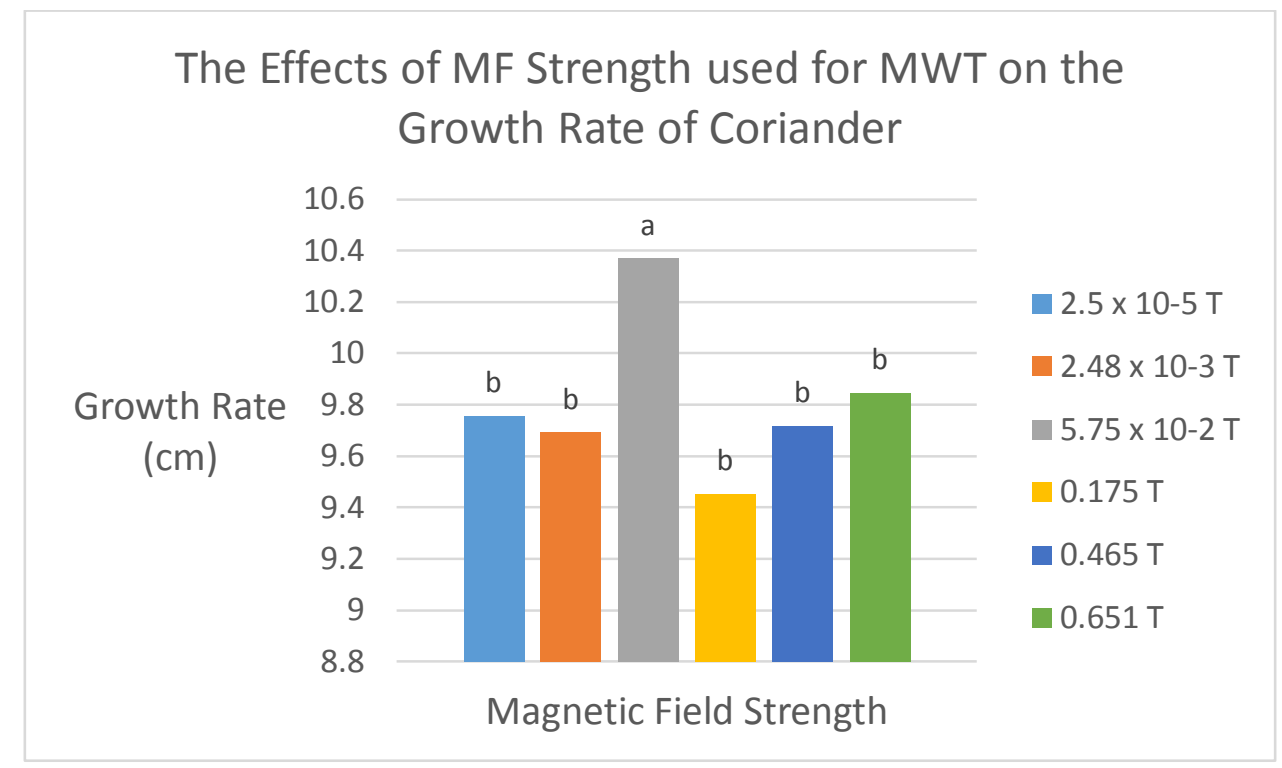

Different letters between treatments differ statistically for $p<0.05$ according to Tukey's test

Figure 4. Growth rate of coriander against different MF strengths

The ceramic (Bar 20) had the highest growth rate with significant differences compared to the other treatments including the control (Figure 5). A probable explanation for this might be that the magnet in this case was a ceramic magnet which has a different composition from that of $\mathrm{NdFeB}$ and these magnets have lower MF strength than NdFeB magnets. This being the case then the ceramic MF needed more time for it to induce changes in the structure of normal water and since some of these structure changes are necessary for a positive change in growth rate then the 10 minute exposure time didn't incur enough changes to significantly increase growth rate.

Both NdFeB (Disc 20) and NdFeB (Bar 20) had the lowest growth rate with significant differences when compared to the other treatments.

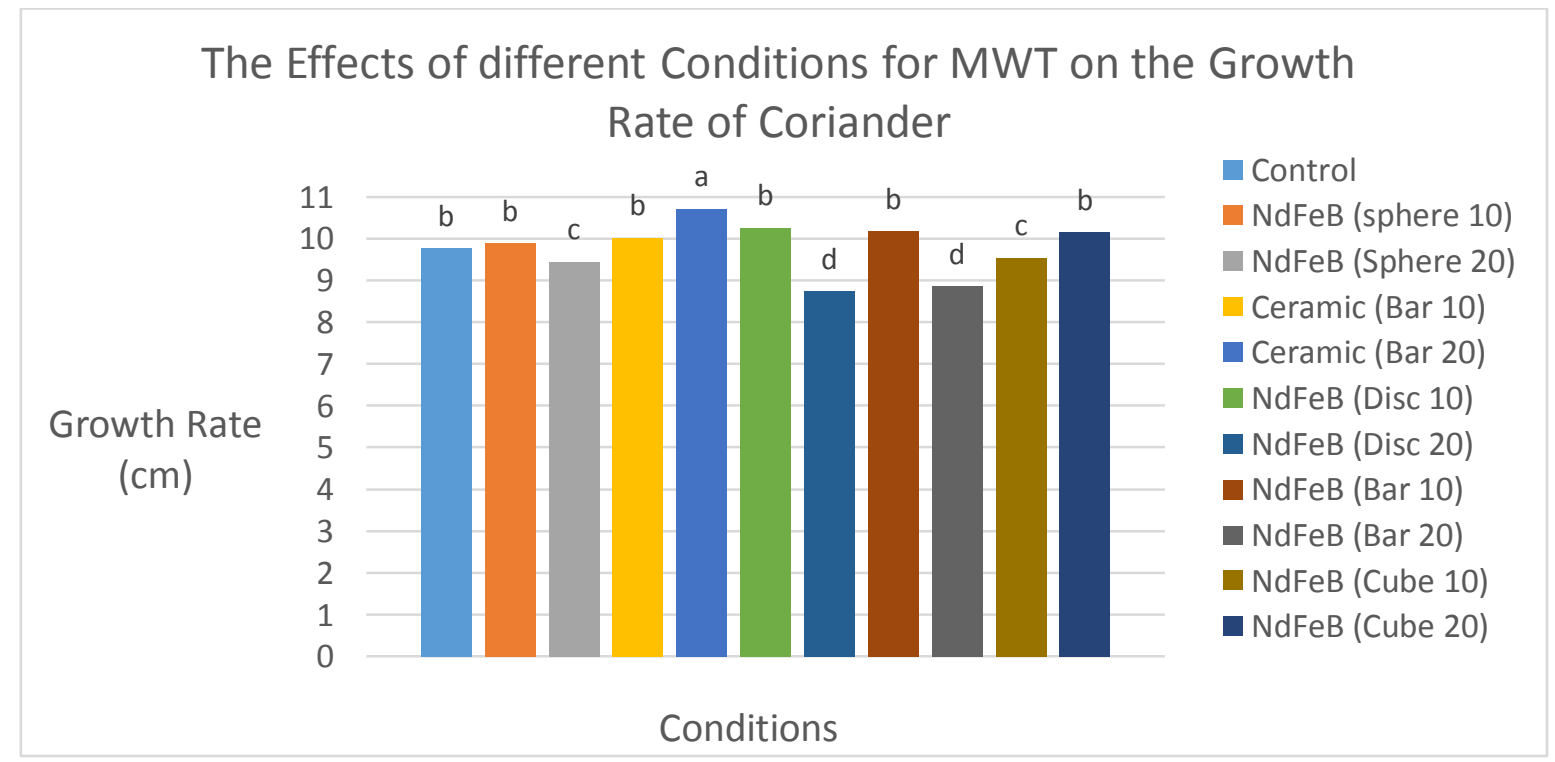

Different letters between treatments differ statistically for $p<0.05$ according to Tukey's test

Figure 5. Growth rate of coriander against different conditions

For this research it can be said that MW had no significant effect on the germination of coriander. One of the reasons that may have led to this result is that the effects of MW is strongly species and genotype dependent. Research has shown that MW does affect germination rate in other species of plants such as wheat cultivars where the seeds were dipped and imbibed in MW exposed to $0.3 \mathrm{~T}$. These treatments had an increase in germination percentage of $9 \%$ to $30 \%$ higher in seven cultivars, but had a 
decrease in the germination rate of the remaining cultivars [3]. Nonetheless, the results from this research always reveal that the growth rate is affected by the type, shape and strength of the magnets used to produce magnetic water.

\section{CONCLUSION}

MWT showed no significant difference in the germination rate of coriander, but it did significantly affect the growth rate. The highest growth rate was obtained by Ceramic (Bar 20). Results revealed that exposure time, type of magnet and MF strength used in MWT influenced the growth rate of coriander.

\section{ACKNOWLEDGEMENT}

We would like to thank the Protected Areas Conservation Trust (PACT) for providing financial assistance for the successful completion of this research.

\section{REFERENCE}

1. Nisticò, R. (2017). Magnetic materials and water treatments for a sustainable future. Research on Chemical Intermediates, 43(12), 6911-6949.

2. Eşitken, A., \& Turan, M. (2004). Alternating magnetic field effects on yield and plant nutrient element composition of strawberry (Fragaria x ananassa cv. camarosa). Acta Agriculturae Scandinavica, Section B - Soil \& Plant Science, 54(3), 135-139.

3. Teixeira da Silva, J. \& Dobránszki, J. (2014). Impact of magnetic water on plant growth. Environmental and Experimental Biology, 12, 137 -142.

4. Pang, F. \& Deng, B. (2008). Investigation of changes in properties of water under the action of a magnetic field. Sci. China Ser. G: Phys. Mech. Astro. 51: 1621-1632.

5. Ali, Y., Samaneh, R., \& Kavakebian, F. (2014). Applications of Magnetic Water Technology in Farming and Agriculture Development: A Review of Recent Advances. Current World Environment, 9(3), 695-703.

6. Camacho, J. \& Sosa, V. (2013). Alternative method to calculate the magnetic field of permanent magnets with azimuthal symmetry. Revista Mexicana de Fisica, 59: 8-17.

7. Dhawi, F. (2014). Why Magnetic Fields are used to Enhance a Plant's Growth and Productivity? Annual Research \& Review in Biology, 4(6), 886-896.

8. Matwijczuk, A., Kornarzyński, K., \& Pietruszewski, S. (2012). Effect of magnetic field on seed germination and seedling growth of sunflower. International Agrophysics, 26(3), 271-278.

9. Ulgen, C., Yıldırım, A. B., \& Turker, A. U. (2017). Effect of Magnetic Field Treatments on Seed Germination of Melissa officinalis L. International Journal of Secondary Metabolite, 63-69.

10. Podsiadlo, C. \& Skorupa, B. (2017). Impact of magnetized water on germination energy of seeds and weight of garden savory (Satureja hortensis l.), buckwheat (Fagopyrum esculentum l.), yellow lupine (Lupinus luteus l.) and winter rape (Brassica napus l.) seedlings. Polish Academy of Sciences

*Corresponding author: Email: ddaniels@ub.edu.bz 